\title{
EVALUACIÓN PARTICIPATIVA DEL EMPODERAMIENTO JUVENIL CON GRUPOS DE JÓVENES. ANÁLISIS DE CASOS
}

\author{
PARTICIPATORY EVALUATION OF YOUTH EMPOWERMENT \\ IN YOUTH GROUPS. CASES ANALYSIS
AVALIAÇÃO PARTICIPATIVA DA CAPACITAÇÃO JUVENIL COM GRUPOS DE JOVENS. ANÁLISES DE CASOS

\author{
Xavier ÚCAR*, Anna PLANAS**, Ana NOVELLA*** \\ \& M. Pilar RODRIGO MORICHE**** \\ *Universidad Autónoma de Barcelona (UAB), ** Universitat de Girona, \\ *** Universidad de Barcelona, ${ }^{* * * *}$ Universidad Autónoma de Madrid (UAM)
}

PALABRAS CLAVES:

pedagogía social

evaluación

participativa

empoderamiento juvenil

investigación

evaluativa

\footnotetext{
Fecha de recepción del artículo: 24.II.2017

Fecha de revisión del artículo: 15.111.2017

Fecha de aceptación final: 17.IV.2017
}

RESUMEN: Las interpretaciones que realizan los jóvenes sobre sus propias realidades son clave para comprender sus acciones y comportamientos. También para adaptar los proyectos y programas socioeducativos a las nuevas dinámicas sociales. Este artículo se centra en el análisis de dichas interpretaciones. ¿Qué entienden los jóvenes por empoderamiento juvenil? ¿Qué indicadores específicos consideran que permiten identificarlo? ¿En qué espacios, momentos y procesos piensan que se produce? Para dar respuesta a estos interrogantes se realizaron 4 procesos de Evaluación Participativa con 42 jóvenes, de entre 14 y 25 años, en 4 ciudades españolas. La muestra de jóvenes que configura los grupos de evaluación participativa en cada uno de los casos es intencional. El artículo contextualiza los casos, presenta la metodología seguida en el desarrollo de la evaluación participativa y aporta los resultados principales de cada caso. Los resultados muestran que los jóvenes de los casos analizados relacionan el empoderamiento con algún tipo de enriquecimiento o mejora de tipo personal o grupal normalmente asociado a un proceso personal, aunque se reconoce la influencia social. Los indicadores considerados más relevantes para el empoderamiento juvenil son la autonomía y la autoestima. Los jóvenes relacionan el empoderamiento juvenil con espacios vinculados al ámbito familiar, escolar y con las amistades. Según las características de los grupos aparecen también como significativos; el espacio extracurricular, la calle y el mundo asociativo. Los procesos de empoderamiento juvenil tienen que ver con vivencias de superación que hicieron que los jóvenes tuvieran percepción de éxito, de superación, de ser importantes para alguien o, por último, de sentir bienestar. La evaluación participativa ha resultado ser una estrategia de intervención socioeducativa muy adecuada para ayudar a las personas jóvenes participantes a construir perspectivas diferentes sobre sus propias vidas.

CONTACTAR CON LOS AUTORES: Xavier Úcar. Dpto. Pedagogía Sistemática y Social. Universidad Autónoma de Barcelona (España) Edifici G6 Campus de la UAB. 08193 Bellaterra (Cerdanyola del Vallès). Universidad Autónoma de Barcelona (UAB). Xavier.ucar@uab.cat. 


\begin{tabular}{|c|c|}
\hline $\begin{array}{l}\text { KEY WORDS: } \\
\text { social pedagogy } \\
\text { participatory } \\
\quad \text { evaluation } \\
\text { youth empowerment } \\
\text { evaluative research }\end{array}$ & $\begin{array}{l}\text { ABSTRACT: Interpretations made by young people about their own realities are key to un- } \\
\text { derstand their actions and behaviours. Also for adapting to new social dynamics socio-ed- } \\
\text { ucational programmes and projects. This article focuses on the analysis of those interpre- } \\
\text { tations. What do youth understand by youth empowerment? Which specific indicators do } \\
\text { they consider to identify it? In which spaces, moments and processes do they think that it } \\
\text { could happen? In order to respond to these questions, } 4 \text { evaluation participatory process- } \\
\text { es with } 42 \text { young people from } 14 \text { to } 25 \text { years old in } 4 \text { Spanish cities were developed. The } \\
\text { sample that sets the participatory evaluation groups for every case is intentional. This paper } \\
\text { contextualizes cases, presenting the followed methodology in the participatory evaluation's } \\
\text { development and provides the main findings of each case. The results show that youth from } \\
\text { the analyzed cases consider the empowerment to be related with a personal or group en- } \\
\text { richment or improvement that is normally associated with a personal process, although its } \\
\text { social influence is recognized. The most relevant indicators for youth empowerment are au- } \\
\text { tonomy and self-esteem. Young people relate youth empowerment to spaces linked to family, } \\
\text { school and friends. The extracurricular space, the street and the associative world appear to } \\
\text { be significant to some of them. Youth empowerment processes have to do with overcoming } \\
\text { experiences that made young people to have a perception of success, to overcome, being } \\
\text { important to someone or eventually, to feel well being. Participatory evaluation has proved } \\
\text { to be a very adequate socio-educational intervention strategy to help participants building } \\
\text { different perspectives on their own lives. }\end{array}$ \\
\hline $\begin{array}{l}\text { PALAVRAS-CHAVE: } \\
\text { pedagogia social } \\
\text { avaliação } \\
\text { participativa } \\
\text { capacitação juvenil } \\
\text { investigação } \\
\text { avaliativa }\end{array}$ & $\begin{array}{l}\text { RESUMO: As interpretaçães que os jovens realizam sobre as suas próprias realidades são } \\
\text { fundamentais para compreender as suas ações e comportamentos. Também para adaptar } \\
\text { os projetos e programas socioeducativos às novas dinâmicas sociais. Este artigo centra-se } \\
\text { na análise dessas interpretações. O que entendem os jovens por capacitação juvenil? Que } \\
\text { indicadores específicos consideram que permitem identificá-lo? Em que espaços, momentos } \\
\text { e processos pensam que se produz? Para dar resposta a estas questões, realizaram-se qua- } \\
\text { tro processos de avaliação participativa com } 42 \text { jovens, entre os } 14 \text { e os } 25 \text { anos, em quatro } \\
\text { cidades espanholas. A amostra de jovens que configura os grupos de avaliação participativa } \\
\text { em cada um dos casos é intencional. O artigo contextualiza os casos, apresenta a metodolo- } \\
\text { gia seguida no desenvolvimento da avaliação participativa e indica os resultados principais } \\
\text { de cada caso. Os resultados demonstram que os jovens dos casos analisados relacionam a } \\
\text { capacitação com algum tipo de enriquecimento ou melhoria de tipo pessoal ou de grupo nor- } \\
\text { malmente associado a um processo pessoal, apesar de se reconhecer a influência social. Os } \\
\text { indicadores considerados mais relevantes para a capacitação juvenil são a autonomia e a } \\
\text { autoestima. Os jovens relacionam a capacitação juvenil com espaços vinculados ao âmbito } \\
\text { familiar e escolar e com as amizades. De acordo com as características dos grupos, apare- } \\
\text { cem também como significativos o espaço extracurricular, a rua e o mundo associativo. Os } \\
\text { processos de capacitação juvenil têm a ver com vivências de superação que fizeram com que } \\
\text { os jovens tivessem perceção do sucesso, de superação, de serem importantes para alguém } \\
\text { e, por último, de sentirem bem-estar. A avaliação participativa revelou-se uma estratégia de } \\
\text { intervenção socioeducativa muito adequada para ajudar as pessoas jovens participantes a } \\
\text { criar perspetivas diferentes sobre as suas próprias vidas. }\end{array}$ \\
\hline
\end{tabular}

\section{Introducción}

En los últimos años la juventud aparece cada vez más como una categoría de estudio de las ciencias sociales. Entender cómo interpretan los jóvenes sus realidades puede ser un conocimiento clave para comprender sus acciones y comportamientos. También para acompañarlos en la construcción de su presente y futuro como miembros activos de la comunidad. Esta es una tarea a la que los investigadores y profesionales de lo social y específicamente los de la pedagogía social, se han estado dedicando las dos últimas décadas (Soler, Pascual, De Juanas, Novella \& Llena, 2016).

La investigación que presentamos se enmarca en el "Proyecto HEBE. El empoderamiento de los jóvenes: Análisis de los momentos, espacios y procesos que contribuyen al empoderamiento juvenil", financiado en la convocatoria nacional de I+D+I (Ref.: EDU2013-42979-R). Este proyecto pretende conocer $y$ analizar los mecanismos $y$ procesos implicados en el empoderamiento de los jóvenes para orientar las políticas de juventud y el trabajo en el ámbito juvenil.

Una de las estrategias metodológicas de investigación utilizadas en este proyecto para generar conocimiento sobre el empoderamiento juvenil es la evaluación participativa. Su pretensión es que sean los propios jóvenes quienes llenen el concepto de contenido. Las preguntas que sustentan esta investigación son: ¿Qué entienden los jóvenes por empoderamiento juvenil? ¿Qué indicadores específicos consideran que permiten identificarlo? ¿En qué espacios, momentos y procesos piensan que se produce el empoderamiento?

Para dar respuesta a estas preguntas, hemos organizado este texto en cuatro apartados. El primero plantea las bases teóricas que fundamentan 
el uso de la evaluación participativa como metodología de investigación. A continuación, se explica la selección de los casos de análisis y la metodología de evaluación participativa. En el tercer apartado, se presenta la contextualización y los principales resultados obtenidos en cada caso. Para acabar, se elaboran las conclusiones generales del proceso de evaluación participativa del empoderamiento juvenil desarrolladas con los grupos de jóvenes.

\section{Evaluación participativa: conceptualización y consideraciones metodológicas}

La evaluación participativa es una estrategia y una práctica de evaluación que aparece en la década de los 90 del siglo pasado. No presenta un enfoque homogéneo en evaluación, sino que agrupa un conjunto de planteamientos y propuestas evaluadoras caracterizadas como "enfoques de evaluación orientados a la participación” (Núñez, Crespo, Úcar \& Llena, 2014). Núñez (2015) apunta que dichos enfoques son el resultado de la confluencia de tres líneas de investigación e intervención: (a) los modelos de cuarta generación de evaluación (Guba \& Lincoln, 1989); (b) la investigación-acción participativa (Suárez-Balcazar, Orellana-Damacela, Portillo, Sharma \& Lanum, 2003); y (c) la teoría del empoderamiento (Suárez-Balcazar et al., 2003).

La evaluación participativa es, según Cousins (2003), un proceso evaluador en el que personas entrenadas en metodologías y técnicas evaluativas desarrollan actividades de evaluación con otras personas no capacitadas en aquellas prácticas. Lo que esta metodología pretende es que personas expertas y no expertas en evaluación desarrollen conjuntamente las actividades necesarias para generar un conocimiento evaluativo compartido sobre las acciones y proyectos en los que participan, o cuyos resultados pueden afectarles.

Tres son las características fundamentales que, desde el punto de vista de Cousins \& Withmore (1998), delimitan lo que es una evaluación participativa:

A) El control técnico de la evaluación tiene que ser compartido por el mayor número de personas implicadas. Esto significa que los expertos en evaluación toman decisiones sobre el diseño y desarrollo del proceso de evaluación de manera compartida con el resto de los participantes (stakeholders).

B) La evaluación participativa tiene que asegurar la diversidad de los agentes implicados. A mayor diversidad de agentes implicados, más participativa será una evaluación concreta (Daigneault \& Jacob, 2009).
C) La evaluación participativa tiene que asegurar, por último, la extensión de la implicación de los agentes. Esto significa que la participación de los agentes y la toma de decisiones sobre el desarrollo de la evaluación debe producirse en todas las fases del proceso. Hay que negociar aspectos como los objetivos y el contenido de la evaluación; la temporización del proceso; las técnicas e instrumentos de recogida y análisis de datos; la presentación de los resultados de la evaluación, etc.

Estas características de la evaluación participativa se conjugan con los criterios que, según Weaver \& Cousins (2004), justifican la utilidad y funcionalidad de dicha estrategia de evaluación. Desde su perspectiva la evaluación participativa es útil porque cumple los siguientes criterios: (1) Es pragmática: orientada a la resolución de problemas; (2) Es política: alineada con la justicia social; y, por último, (3) Es epistemológica: permite la validación del conocimiento no experto. Úcar, Heras \& Soler (2014) añaden un cuarto criterio: la utilidad o justificación que denominan pedagógica o empoderadora; encaminada, en este caso, a la adquisición de recursos personales y comunitarios que empoderan a las personas implicadas. Es una estrategia socio-pedagógica que facilita el aprendizaje y la adquisición de recursos a las personas involucradas.

Núñez y otros (2014) analizan los principales modelos de evaluación orientados a la participación: (1) la evaluación colaborativa; (2) la evaluación participativa práctica; (3) la evaluación participativa transformadora; y, por último, (4) la evaluación empoderadora. Del análisis concluyen que los dos últimos modelos de evaluación participativa se ajustan mejor a los planteamientos de la acción comunitaria y del trabajo con jóvenes en el marco de la pedagogía social. Coincidimos con ellos en que todos los modelos de evaluación orientados a la participación se constituyen como procesos educativos mediante los cuales las personas y las comunidades pueden aprender y empoderarse. La evaluación participativa en la que encuadramos nuestras acciones es aquella que nos permite cambiar y cambiarnos para, a través de este mismo proceso, adquirir nuevos poderes que nos permitan transformar las realidades que estamos viviendo (Núñez y otros, 2014, p. 97).

\section{Selección de los casos}

El proceso de investigación evaluativa se inicia con la selección de los casos en los que se va a desarrollar la evaluación participativa. Definimos el caso como un grupo de jóvenes ubicado en una comunidad institucional o territorial. Cada grupo 
se constituye como un caso que, siguiendo la tipología de Coller (2000), es caracterizado como: (1) Procesual: proceso de evaluación participativa que se desarrolla en grupos de jóvenes configurados en marcos institucionales de base territorial; (2) Específico y único: cada caso es intencionalmente seleccionado por estar vinculado a un contexto institucional; (3) Contemporáneo: se refiere a fenómenos que se desarrollan en la actualidad; y, finalmente, (4) Analítico: se estudia cómo se desarrollan los fenómenos en cada caso para analizarlos y extraer conclusiones.

Los criterios para la selección de los jóvenes de cada caso son: emancipados/no emancipados; trabajando/no trabajando; paridad de género; extranjeros/autóctonos; y trayectoria universitaria/ no universitaria. Dada la dificultad de implicar a los jóvenes en el proceso, se decide que los criterios de selección de jóvenes, sean cumplidos por el conjunto de los casos seleccionados y no específicamente por cada uno de ellos.

La muestra de jóvenes que va a configurar los grupos de evaluación participativa es una muestra intencional que obedece tanto a la predisposición y disponibilidad para participar, de los jóvenes y de las instituciones o entidades que los amparan, como a las posibilidades de acceso del equipo de investigación a dichos grupos. Los cuatro grupos de jóvenes que aceptan desarrollar el proceso de evaluación participativa son:

1. "Casal de Joves" de Badia del Vallés (Barcelona)

2. "Els Químics Espai Jove" de Girona

3. "Consell de la Joventut de Barcelona" (CJB)

4. "Grupo asociación Norte joven Vallecas" (Madrid)

Se trabaja con 42 jóvenes ( 22 chicas y 20 chicos) de edades comprendidas entre los 14 y los 25 años (tabla 1).

Tabla 1. Configuración de los grupos de jóvenes de los casos

\begin{tabular}{|l|c|c|c|c|}
\hline & $\begin{array}{c}\text { Intervalo } \\
\text { de edad }\end{array}$ & Total & Chicas & Chicos \\
\hline $\begin{array}{l}\text { Grupo 1 } \\
\text { (Badia del Vallés) }\end{array}$ & 14 y 20 años & 15 & 8 & 7 \\
\hline $\begin{array}{l}\text { Grupo 2 } \\
\text { (Girona) }\end{array}$ & 17 y 25 años & 8 & 3 & 5 \\
\hline $\begin{array}{l}\text { Grupo 3 } \\
\text { (Barcelona) }\end{array}$ & 19 y 24 años & 10 & 6 & 4 \\
\hline $\begin{array}{l}\text { Grupo 4 } \\
\text { (Madrid) }\end{array}$ & 16 y 22 años & 9 & 5 & 4 \\
\hline
\end{tabular}

\section{Objetivos y metodología de la evaluación participativa con los grupos de jóvenes}

El planteamiento de partida en cada caso es que dos miembros del equipo de investigación y un facilitador, generalmente un educador social de la propia institución donde se desarrolla el proceso, son los expertos en evaluación que van a trabajar con el grupo de jóvenes; que son los no expertos en evaluación. Ambos constituyen el equipo de evaluación participativa del empoderamiento juvenil en cada uno de los ámbitos institucionales seleccionados como caso de análisis.

Dado que este proceso de evaluación participativa con jóvenes forma parte de una investigación más amplia, los objetivos a conseguir con dicho proceso habían sido previamente definidos en el marco de aquella investigación. Que estuvieran previamente definidos no exime al equipo de investigación de la necesidad de consensuarlos y adaptarlos, reformularlos o eliminarlos, si así se decidiera, en cada grupo de evaluación participativa juvenil.

Estos son los objetivos inicialmente planteados:

a) Construir un significado sobre el concepto de empoderamiento juvenil.

b) Evaluar el concepto de empoderamiento juvenil elaborado por el grupo de investigación.

c) Evaluar una batería de indicadores de empoderamiento juvenil.

d) Relacionar los indicadores de empoderamiento juvenil con los espacios, los momentos y los procesos en los que se desarrolla la vida cotidiana de los jóvenes.

Para lograr estos objetivos se parte de unas bases documentales, previamente elaboradas por el grupo de investigación, que se utilizan como objetos específicos de evaluación. Son dos $^{2}$ :

a) Concepto de empoderamiento juvenil construido por el equipo de investigación ${ }^{3}$.

b) Batería de indicadores de empoderamiento juvenil elaborada por el grupo de investigación ${ }^{4}$.

Se parte de la base de que cada grupo de evaluación participativa es autónomo y soberano para decidir el desarrollo y las características del proceso de evaluación participativa. Aun así, se diseña una estructura metodológica como guía para el desarrollo de las sesiones de evaluación participativa que también ha de ser validada por cada grupo.

Como se puede observar en la tabla 2, las sesiones 2 y 3 fueron dedicadas a lo que denominamos "construcción del criterio". Aunque el 
término "empoderamiento" se ha popularizado en los últimos años pensamos que podía resultar extraño para algunos de los jóvenes participantes. Eso nos llevó a proponer dos sesiones iniciales de trabajo donde los jóvenes buscaran por su cuenta y debatieran sobre el significado de estar "empoderado". Eso les permitió construir sus propios referentes conceptuales desde los que evaluar el concepto propuesto por el grupo de investigación.

Tabla 2. Estructura metodológica para el desarrollo de las sesiones de evaluación participativa

\begin{tabular}{|c|c|}
\hline Sesiones & Contenido \\
\hline $\begin{array}{l}\text { Sesión O: } \\
\text { Formación de los facilitadores }\end{array}$ & $\begin{array}{l}\text { - Sesión de formación } \\
\text { - Equipo de investigación y facilitadores de cada grupo de } \\
\text { jóvenes }\end{array}$ \\
\hline $\begin{array}{l}\text { Sesión 1: } \\
\text { Presentación del proyecto a los grupos de jóvenes }\end{array}$ & $\begin{array}{l}\text { - Presentación del proyecto a los jóvenes } \\
\text { - Análisis y validación del proceso de evaluación } \\
\text { participativa propuesto }\end{array}$ \\
\hline $\begin{array}{l}\text { Sesiones } 2 \text { y } 3 \text { : } \\
\text { Construcción del criterio en los grupos de jóvenes } 5 \text { e } \\
\text { identificación de espacios, momentos y procesos de } \\
\text { empoderamiento juvenil }\end{array}$ & $\begin{array}{l}\text { - Los grupos elaboran su propio concepto de } \\
\text { empoderamiento y piensan cómo, dónde y cuándo se } \\
\text { empoderan }\end{array}$ \\
\hline $\begin{array}{l}\text { Sesión 4: } \\
\text { Evaluación del concepto de empoderamiento juvenil }\end{array}$ & - Dinámicas de trabajo para evaluar el concepto \\
\hline $\begin{array}{l}\text { Sesión 5: } \\
\text { Evaluación de los indicadores de empoderamiento juvenil }\end{array}$ & $\begin{array}{l}\text { - Dinámicas de trabajo para evaluar la batería de } \\
\text { indicadores }\end{array}$ \\
\hline $\begin{array}{l}\text { Sesión 6: } \\
\text { Cierre del proceso }\end{array}$ & $\begin{array}{l}\text { - Los jóvenes debaten sobre lo aprendido del proceso y lo } \\
\text { evalúan }\end{array}$ \\
\hline
\end{tabular}

Esta fue la propuesta de trabajo que cada uno de los equipos de evaluación participativa adaptó a sus particulares intereses y características. La estructura metodológica resultante en cada uno de los casos puede verse en el Tabla 3.

\begin{tabular}{|c|c|c|l|l|}
\hline \multicolumn{5}{|c|}{ Tabla 3. Estructura metodológica del desarrollo de las sesiones } \\
de evaluación participativa en cada caso
\end{tabular}




\begin{tabular}{|c|l|l|l|l|}
\hline 5 & $\begin{array}{l}\text { - Evaluación batería de } \\
\text { indicadores } \\
\text { Cierre del proceso }\end{array}$ & $\begin{array}{l}\text { - Evaluación batería de } \\
\text { indicadores }\end{array}$ & $\begin{array}{l}\text { - Identificación de } \\
\text { espacios, momentos y } \\
\text { procesos (1) }\end{array}$ & $\begin{array}{l}\text { Evaluación batería de } \\
\text { indicadores (2) }\end{array}$ \\
\hline 6 & - Cierre del proceso & $\begin{array}{l}\text { - Identificación de } \\
\text { espacios, momentos y } \\
\text { procesos (2) } \\
\text { Cierre del proceso }\end{array}$ & Cierre del proceso \\
\hline
\end{tabular}

En lo que se refiere a la temporalidad de los procesos en cada caso, hay que señalar que se desarrollaron entre los meses de enero y junio de 2016. La recomendación inicial general era hacer una sesión de evaluación participativa cada semana, pero la frecuencia fue finalmente decidida por cada equipo.

\section{Análisis de los casos}

En este apartado se presenta una breve contextualización y los principales resultados de la evaluación participativa obtenidos de cada caso.

\subsection{Caso 1: "Casal de Joves" de Badia del Vallés}

Badia del Vallés es un pueblo cercano a Barcelona que tiene una de las densidades de población más elevadas de Cataluña. El nivel sociocultural es medio/bajo.

La evaluación participativa se ha desarrollado en un equipamiento municipal, el Casal de Joves, al que los jóvenes participantes suelen acudir por las tardes después de clase. Buena parte de estos jóvenes no se conocían previamente. El grupo ha estado configurado por 17 jóvenes; todos escolarizados (ESO/Bachillerato/Ciclos formativos).

\subsubsection{Resultados de la evaluación participativa}

Los jóvenes conectan la idea de aprender con tener poder, lo que, desde su punto de vista, les lleva a estar o sentirse empoderados. Además, afirman que el empoderamiento surge ante situaciones en las que deben salir adelante por sí mismos o ayudar a otras personas. Los términos que, a lo largo del proceso de construcción del criterio de evaluación, relacionan con el empoderamiento son: poder, fuerza, mejora personal, responsabilidad, liderazgo, autocontrol y participación.

El grupo de jóvenes valida claramente la definición de empoderamiento elaborada por el grupo de investigación y la utiliza para reformular la definición que ellos habían elaborado en el proceso de construcción del criterio. De hecho, incorporan a su definición elementos de los investigadores y acaban definiendo el empoderamiento como
"Poder y capacidad de decisiones, situaciones, fuerzas sobre uno mismo mediante la interacción con un grupo de personas y que lleva una mejora". Consideran que, aunque es posible ayudar a las personas a empoderarse, el empoderamiento es un proceso individual.

La validación de los indicadores se realiza a través de representaciones teatrales. A partir de ellas todos los indicadores de la batería se entienden y validan, a excepción de "metaprendizaje", que les resulta difícil de entender. El grupo de jóvenes identifica el indicador "Autoestima" como el más importante en relación al empoderamiento juvenil. También consideran importantes los indicadores de "autonomía" y "responsabilidad". Todos los jóvenes coinciden en la necesidad de incorporar un nuevo indicador: "liderazgo".

Los jóvenes de Badia piensan que el empoderamiento juvenil se relaciona con los espacios directamente vinculados a las vivencias y experiencias de los jóvenes; primero, en su entorno familiar y, segundo, en el ámbito social-escolar y con su grupo de iguales. En el ámbito familiar, los jóvenes dicen sentirse empoderados cuando logran afrontar situaciones difíciles, con referentes como sus padres, sus hermanos o sus abuelos. En el segundo ámbito se refieren a los espacios extracurriculares como lugares estratégicos para el desarrollo de sus habilidades interpersonales. En ellos pueden llegar a establecer vínculos de amistad con sus iguales y también posibilitan que descubran y pongan en práctica sus intereses personales, por ejemplo, la música y el deporte. Sin embargo, también afirman haber experimentado momentos en los cuales han tenido que seguir adelante pese a las dificultades. En este caso, fue la confianza en ellos mismos y los referentes externos lo que les ayudó a continuar.

Todos los participantes consideran que el proceso de evaluación participativa se ha desarrollado de manera muy eficaz y satisfactoria. Los jóvenes afirman que han aprendido un concepto nuevo: "empoderamiento". También dicen que han aprendido a trabajar en equipo, "a ayudarnos unos a otros" y a mejorar sus relaciones interpersonales. 


\subsection{Caso 2: "Els Químics Espai Jove" de Girona}

El proceso se inicia con la oportunidad de coordinar la evaluación participativa con la evaluación del equipamiento Els Químics Espai Jove que el Ayuntamiento de Girona pretendía desarrollar.

Els Químics Espai Jove se creó en 2011, y es un espacio de encuentro destinado a jóvenes entre 13 y 20 años. Aunque está especializado en la orientación académica, ofrece también servicios y actividades que responden a las inquietudes y necesidades de los jóvenes.

Los 8 jóvenes participantes en el proceso de evaluación participativa tienen entre 17 y 25 años, y han sido usuarios de Els Químics Espai Jove durante los primeros años de vida del equipamiento.

\subsubsection{Resultados de la evaluación participativa}

Aunque el término "empoderamiento" es nuevo para estos jóvenes, lo van integrando paulatinamente durante el proceso de trabajo. Para ello recurren constantemente a la utilización de ejemplos personales que reconocen como empoderadores y que en gran medida están vinculados a procesos de toma de decisiones. Los jóvenes asocian empoderamiento con tener iniciativa, correr riesgos, escoger, o salir de la zona de confort (Planas, Turon, Páez de la Torre, Bartomeus, Arumí, 2016). También identifican dos grandes aspectos para el empoderamiento. Por un lado, reconocen las capacidades personales o aspectos que dependen de uno mismo para estar empoderado: autoconfianza, comunicación, motivación, autoestima, seguridad, empatía, etc. Por otro, los condicionantes externos: la sociedad de pertenencia, la determinación social y la situación personal que se vive en un determinado momento. A partir de esto reflexionan sobre la importancia de tener conciencia de la realidad, de entender los diferentes puntos de vista, y de ser consciente de las manipulaciones, para estar empoderado. A todo ello añaden que estar empoderado "en el buen sentido" requiere también tener ética, empatía, o respeto hacia los otros.

En relación con la definición del término presentada por el equipo de investigación, los jóvenes resaltan la importancia de las condiciones del entorno para el empoderamiento juvenil, así como las potencialidades/capacidades innatas que cada uno tiene.

La validación de los indicadores de empoderamiento se realiza después de un trabajo previo a partir de dinámicas teatrales, con lo que los jóvenes interiorizan estos indicadores de una forma práctica y muy visual. Los jóvenes dan por válidos todos los indicadores propuestos. Para la jerarquización de los indicadores trabajan en dos grupos. La autoestima y la autonomía son los indicadores más importantes para el empoderamiento en ambos grupos. Los otros indicadores adquieren posiciones dispares en los dos grupos. En un grupo resaltan la capacidad crítica, la eficacia, y la participación, dejando en último lugar, identidad y conocimiento comunitario y metaprendizajes. En el otro, destacan la responsabilidad, seguida de identidad y conocimiento comunitario, y capacidad crítica, y sitúan en últimas posiciones, eficacia y participación.

La idea inicial era trabajar los espacios, momentos y procesos de empoderamiento a lo largo de las diferentes sesiones a través de fotos que los jóvenes iban colgando en un grupo de Instagram. Los resultados, sin embargo, no fueron muy productivos, por lo que se cerró el proceso con una dinámica específica: el dibujo de la línea del tiempo. Como espacios clave para el empoderamiento aparecen: la familia, los centros educativos, la calle o plazas y las asociaciones o actividades extraescolares. Incidentes críticos, como la muerte de algún familiar o el traslado de lugar de residencia, también son considerados clave en las vidas de los jóvenes.

La valoración general del proceso de evaluación participativa es positiva. Los jóvenes están satisfechos de los aprendizajes realizados (interiorización del propio concepto de empoderamiento, recursos y aspectos que pueden desarrollar para empoderarse, o identificación de la zona de confort y cómo salir de él) y reconocen haber alcanzado las expectativas que tenían en relación al proceso de evaluación participativa.

Un elemento clave para el desarrollo del proceso ha sido el vínculo que tienen los facilitadores con los jóvenes. Como aspectos limitadores del proceso señalamos la propia dinámica del centro, la irregularidad del proceso de evaluación participativa y la dispersión de los participantes. Todo ello ha dificultado el trabajo de los facilitadores en la dinamización de los jóvenes.

\subsection{Caso 3: "Consell de la Joventut de Barcelona" (CJB)}

El grupo de jóvenes se constituyó a partir de una petición de colaboración al Consejo de la Juventud de Barcelona (CJB). El CJB, como plataforma interasociativa que coordina y representa a las principales entidades juveniles de Barcelona, lanzó la propuesta para constituir un grupo que participara en la investigación.

El grupo está constituido por jóvenes vinculados al mundo asociativo que tienen alguna conexión con el CJB. Es un grupo de 9 participantes 
de entre 19 y 24 años. Todos ellos participan en alguna asociación o entidad. La formación académica de 5 jóvenes es bachillerato y de 4 universitaria. Algunos tienen experiencia laboral de carácter "precario". No todos se conocen.

\subsubsection{Resultados de la evaluación participativa}

El término empoderamiento fue rápidamente aceptado por el grupo. Les resultaba familiar pues lo asociaban al movimiento feminista o comunitario y algunos jóvenes lo equiparaban inicialmente a emancipación.

Los jóvenes asocian empoderamiento con crecimiento personal y toma de decisiones. Lo entienden como una transformación interna; como un proceso personal que también es colectivo y social. Aunque dan más valor a la incidencia del grupo, asociación u organización. Según este grupo, el empoderamiento se produce en dos esferas: interna y colectiva. Generan entre ellos un debate sobre si es una vía, un producto, un resultado o una vivencia personal. Buena parte de las deliberaciones se organizaron alrededor de esta diferenciación que también relacionaron con el resto de términos identificados. Se refieren al empoderamiento colectivo social, que está vinculado a la participación, la reivindicación, los movimientos de lucha y el romper con las normas.

En la presentación de los indicadores hubo que clarificar dos. Por un lado, el indicador metaprendizajes, que fue ejemplificado para ayudar a comprenderlo $y$, por otro, el indicador identidad y conocimiento comunitario, sobre el que afirmaron que no permite diferenciar si la identidad es individual o comunitaria.

Para jerarquizar los indicadores se organizaron en dos grupos. Entre los grupos no hubo coincidencia en los indicadores más importantes. De hecho, el indicador que un grupo valora más es muy poco valorado en el otro y viceversa (autoestima e identidad y conocimiento comunitario). Coinciden en los indicadores centrales (capacidad crítica, autonomía, responsabilidad y trabajo en equipo) y en el poco valor que asignan a eficacia. También difieren en el valor que asignan a participación.

En la evaluación de los espacios, momentos y procesos les resultó más fácil identificar momentos que espacios y procesos. Se reconocen empoderados, pero tienen dificultades para identificar el proceso por el que se empoderan. Debaten sobre si el empoderamiento es el inicio de un proceso o su culminación. También sobre si se está ya empoderado antes del momento o experiencia concreta de empoderamiento.

El empoderamiento se inicia en edades tempranas con hechos cotidianos; con la asignación de responsabilidades u oportunidades de decidir. Asocian el empoderamiento a momentos y procesos vinculados a la toma de decisiones de forma autónoma: a moverse y desplazarse con autonomía; a tener responsabilidades, ser elegido referente y reconocerse influyente; a decir no, llevar la contraria o ir contracorriente; a trazar su propia identidad, su yo diferenciado de otros, salirse de aquello socialmente esperado o deseado; a hablar delante de un grupo de gente, hacer algo o manifestar una opinión; a liderar procesos colectivos de transformación, constituir asociación o promover un movimiento de defensa de unos derechos o de un estilo de vida; a alcanzar retos y sueños a partir del esfuerzo personal; $y$, por último, a la perdida de personas significativas, a enfrentarse a los duelos por fallecimiento o por rotura de relación.

La valoración general de la evaluación participativa ha sido muy positiva. El grupo ha sido muy participativo y constructivo con una elevada capacidad reflexiva. Las sesiones les han permitido poder dar forma al concepto y descubrir nuevos componentes y dimensiones Se han reconocido como agentes que promueven oportunidades de empoderamiento para otros dentro de los grupos que lideran en sus asociaciones. Reconocen que se ha producido en ellos un cambio de actitud personal. Afirman que el hecho de pararse a pensar en su trayectoria personal, en cómo el cooperar, compartir ideas e ir más allá de una simple charla, les ha empoderado.

\subsection{Caso 4: "Grupo asociación Norte joven Vallecas"}

El distrito Villa de Vallecas, donde se sitúa la asociación Norte Joven, se caracteriza por tener una de las rentas medias más bajas de la ciudad, por ser uno de los distritos más vulnerables en cuanto al desempleo, y por su elevado número de habitantes de origen extranjero. Vallecas, en general, es una de las zonas con una de las tasas de abandono escolar más alta de Madrid.

La asociación Norte Joven, a través de su programa formativo, ofrece a los jóvenes acceder a los derechos y recursos de la sociedad y al ejercicio de deberes cívicos, ofreciendo otras alternativas de formación para el desarrollo integral de los jóvenes excluidos.

Los jóvenes asisten a una formación específica del centro, un taller profesional de hostelería. El objetivo es lograr la plena inserción en la sociedad a través del conocimiento del oficio y dotarles de competencias para el desarrollo personal y social. Estos jóvenes se encuentran en un momento particularmente vulnerable, con necesidad de 
afecto, de modelos de referencia y de un ambiente enriquecedor.

\subsubsection{Resultados de la evaluación participativa}

El término empoderamiento es un concepto nuevo y extraño para los jóvenes. Desde el principio hay una demanda explícita de información sobre el concepto. A pesar de la resistencia inicial frente al término, el grupo, sin ser muy consciente del proceso, lo ha ido integrando progresivamente.

Dadas sus circunstancias vitales, entienden el empoderamiento como no quedarse estático o atrapado en su situación; lo interpretan como: "salir a buscarse la vida". Habría que matizar que el concepto ha sido utilizado por los jóvenes más como un proceso que como un resultado y más ubicado en las personas que en el contexto. A pesar de todo, los jóvenes consideran que el contexto es una variable importante para abrir/cerrar posibilidades de empoderamiento. También la formación es considerada como un elemento muy importante para empoderarse. La definición del término que se presenta al grupo les ayuda a situarse en el proceso y se dan cuenta de que el empoderamiento formaba parte de sus vidas, pero sin manejar el término exacto.

La presentación de los indicadores de empoderamiento genera dudas puesto que el vocabulario empleado no les resulta familiar. También presentan resistencia por inseguridad frente los términos con los que están formulados los indicadores. Una vez explicados los reconocen con más claridad. En el análisis y evaluación de la batería de indicadores de empoderamiento añaden conceptos y los ponen en relación con los indicadores presentados. Relacionan autoestima con seguridad, fuerza, motivación y energía; autonomía con liderazgo; identidad con valores y responsabilidad. Proponen separar la identidad del conocimiento comunitario, por considerar que uno se refiere a lo singular y el otro a lo colectivo. El grupo ordena los indicadores poniendo la autonomía en primer lugar, seguida del metaprendizaje y autoestima. En tercer lugar, identidad y conocimiento comunitario. A continuación, y al mismo nivel, capacidad crítica, trabajo en equipo y participación. Por último, eficacia.

El grupo de jóvenes interpreta como empoderamiento, el hecho de haber experimentado situaciones de fracaso y haber sobrevivido a determinadas circunstancias críticas en su vida. Polarizan las ideas de "no hacer nada" o "empoderarse".

La evaluación de los espacios, momentos y procesos en los que los jóvenes se empoderan no fue bien desarrollada por el grupo. Las actividades orientadas a generar ejemplos de espacios, momentos y procesos (mural, grupo telegram, actividad en el aula) no han resultado muy productivas, ya que los jóvenes no se han acabado de implicar en ellas.

Sitúan lo más significativo de su aprendizaje en los momentos, procesos y espacios donde son capaces de ver y entender cómo se produce el empoderamiento de manera concreta. Ubican el empoderamiento en el ámbito profesional, en el momento de formación de los jóvenes y en un espacio como el centro educativo.

Además, valoran de forma nítida cuáles son sus capacidades personales para favorecer el empoderamiento y cuáles son las que van a facilitarlo más rápidamente. Ellos se sitúan, en el momento de la evaluación, en un proceso formativo en el que entienden que están empoderándose.

Las dificultades de estos jóvenes, respecto a la capacidad de expresión y fluidez verbal, ha exigido dedicar tiempo a explicar los conceptos de diferentes maneras para lograr que fueran comprendidos por ellos.

La valoración general ha sido muy positiva. Aunque durante el proceso ha habido momentos más productivos que otros, la motivación ha sido alta y los jóvenes se han mostrado agradecidos porque se han sentido empoderados. También porque las sesiones de evaluación participativa les ha generado satisfacción, aprendizajes y reflexión sobre sus propios procesos de empoderamiento.

\section{Conclusiones del proceso de evaluación participativa del empoderamiento juvenil con 4 grupos de jóvenes}

La diferente configuración de los grupos, en lo que se refiere a los niveles culturales, de experiencias personales y de instrucción de los y las jóvenes, hace que la construcción del criterio grupal, en relación al concepto de empoderamiento, se produzca de maneras muy diferenciadas en los cuatro grupos de jóvenes. A pesar de todo se puede afirmar que la creación conjunta de lo que significa e implica el concepto de empoderamiento se produjo en los cuatro grupos. Todos ellos construyeron sus propias conceptualizaciones y definiciones del concepto de empoderamiento juvenil y lo pusieron en relación con sus propias vidas.

Todos los grupos relacionan el empoderamiento con algún tipo de enriquecimiento o de mejora de tipo personal o grupal. Los jóvenes vinculan el empoderamiento con cierta percepción de éxito y/o superación y se conecta a términos como seguridad y confianza en uno mismo. También, el tener fuerza de voluntad y capacidad para imponerse a las dificultades. En los cuatro grupos el empoderamiento se asocia claramente a un 
proceso personal; una transformación interna que pasa por uno mismo, aunque se reconoce la influencia social.

La totalidad de los grupos valida la definición de empoderamiento presentada por el equipo investigador, aunque hay que señalar que incorporan, eliminan o ponen claramente en cuestión algunos de los elementos.

Aunque el proceso de comprensión de los indicadores varía entre grupos, coinciden en la dificultad de comprensión del indicador metaprendizaje. Los cuatro grupos validan la batería de indicadores de empoderamiento presentada por el equipo de investigación. El grupo de Barcelona pone como indicador poco relevante eficacia. Este indicador está situado en último nivel en la jerarquización elaborada por el grupo de Madrid y por uno de los subgrupos de Badia y Girona. El indicador identidad y conocimiento comunitario genera debate en los grupos de Barcelona y Madrid dado que no permite diferenciar entre lo individual y lo comunitario. El grupo de Madrid propone separar identidad asociada a lo personal y conocimiento comunitario a lo colectivo. Todos los grupos realizan una jerarquización de los indicadores. Autonomía y autoestima son considerados, en general, los más importantes. Los grupos de jóvenes proponen incorporar indicadores como liderazgo, confianza, autoconocimiento, seguridad, fuerza, motivación y energía. Todos ellos vinculados al indicador de autoestima.

En todos los grupos el tema de espacios, momentos y procesos de empoderamiento juvenil se trata desde las vivencias y experiencias personales. El empoderamiento juvenil se relaciona con espacios vinculados al ámbito familiar, escolar y con las amistades. En Badia, aparece el espacio extracurricular como significativo porque en él, los jóvenes pueden llegar a establecer vínculos de amistad con sus iguales y también permite que descubran, expresen y pongan en práctica sus intereses personales. También en el grupo de Barcelona aparece este espacio, pero caracterizado como un espacio de decisión y elección autónoma. En Madrid y en Girona los jóvenes identifican, además, un nuevo espacio: la calle. $Y$ en Barcelona aparece, de forma significativa, el espacio asociativo.

Los procesos que aparecen de forma transversal a los espacios identificados tienen que ver con vivencias de superación que hicieron que los jóvenes tuvieran percepción de éxito, de superación, de ser importantes para alguien o, por último, de sentir bienestar. Se identifican procesos de aprendizaje y de crecimiento personal en todos los casos, ya sea por superar los estudios o por tener la valentía de abandonarlos por no ser lo que querían hacer. Se detectan procesos de superación en los que poder tirar adelante frente a situaciones adversas o de fragilidad.

En el caso de Barcelona, algunos procesos de empoderamiento se relacionan con la toma de decisiones en edades tempranas y con la asunción de responsabilidades. Otros procesos citados en los grupos de Madrid y Barcelona tienen que ver con el hecho de independizarse.

Las personas referentes dentro de estos procesos y momentos son familiares (padres, abuelos, tíos, hermanos) y amigos.

Los cuatro grupos coinciden en que el desarrollo del proceso de evaluación participativa ha sido muy satisfactorio y muy bien valorado tanto por los grupos de jóvenes como por los facilitadores. También en el hecho de que los grupos respondieron bien a las dinámicas propuestas y aceptadas. Los facilitadores han sido un elemento clave en el desarrollo de la evaluación participativa.

Todos los grupos afirman haber aprendido durante el proceso. Hablan de dos tipos de aprendizajes: (1) los realizados en relación al propio concepto de empoderamiento, a la multiplicidad de dimensiones que lo configuran y a las formas y maneras en que este se puede manifestar; y (2) los aprendizajes referidos a las formas en que se ven a sí mismos a partir de este concepto. Se puede decir que, en general, miran sus trayectorias vitales de manera diferente distinguiendo aquello que les empodera de aquello que no. Señalan, por último, que el proceso les ha ayudado a poner en valor lo que hacen y les ha dado claves para enfrentarse mejor a sus situaciones vitales.

Todos los elementos anteriores nos permiten concluir que la evaluación participativa, en la forma cómo ha sido utilizada en esta investigación, ha resultado ser una estrategia de intervención socioeducativa muy adecuada para ayudar a las personas jóvenes participantes a construir perspectivas diferentes sobre sus propias vidas. También y como resultado de dichas perspectivas, a dotarlas de instrumentos personales de análisis que les sirvan para introducir, si así lo consideran, cambios en sus vidas. 


\section{Referencias bibliográficas}

Coller, X. (2000). Estudio de casos. Cuaderno No 30. Madrid: Centro de investigaciones sociologicas (CIS).

Cousins, J. B. (2003). Utilization effects of participatory evaluation. En T. Kellaghan, D. L. Stufflebeam \& L. A. Wingate (eds.), International handbook of educational evaluation (pp. 245-265). Boston, MA: Kluwer.

Cousins, J. B., \& Whitmore, E. (1998). Framing Participatory Evaluation. New Directions for Evaluation, 80, 5-23.

Daigneault, P.-M., \& Jacob, S. (2009). Toward Accurate Measurement of Participation: Rethinking the Conceptualization and Operationalization of Participatory Evaluation. American Journal of Evaluation, 30 (3), 330-348.

Guba, E., \& Lincoln, Y. (1989). Fourth generation evaluation. California: Newsbury Park.

Núñez, H. (2015). Evaluación participativa en la acción comunitaria. Aproximaciones teórica y metodológica. Madrid: Popular.

Núñez, H., Crespo, E., Úcar, X. \& Llena, A. (2014). Enfoques de evaluación orientados a la participación en los procesos de acción comunitaria. Pedagogía Social. Revista Interuniversitaria, 24, 79-103. doi: 10.7179/PSRI_2014.24.04.

Planas, A., Turón, N., Páez de la Torre, S., Bartomeus, A., \& Arumí, À. (2016). Evaluación participativa del empoderamiento juvenil. Proceso de evaluación participativa en "Els Químics Espai Jove" de Girona. http://hdl.handle. net/10256/13402

Rodrigo, M. P., Páez, J., \& Arenillas, M. (2016). Evaluación participativa del empoderamiento juvenil. Proceso de evaluación participativa con jóvenes de la "Asociación Norte Joven Vallecas" de Madrid. Depósito Digital de Documentos. Universidad Autónoma de Madrid. http://hdl.handle.net/10486/676599.

Soler, P., Planas, A., Ciraso-Calí, A., \& Ribot-Horas. A. (2014). Empoderamiento en la comunidad. El diseño de un sistema abierto de indicadores a partir de procesos de Evaluación Participativa. Pedagogía Social. Revista Interuniversitaria, 24 49- 77. doi:10.7179/PSRI_2014.24.03.

Soler, P., Pascual, B., De Juanas, A., Novella, A., \& Llena, A. (2016). Reflexiones finales del Congreso Internacional - XXIX Seminario Interuniversitario de Pedagogía Social. Pedagogía Social, Juventud y Transformaciones Sociales. Documento electrónico. Retrieved from http://dugi-doc.udg.edu/bitstream/handle/10256/13325/CongresoPedagogiaSocialXXIX-Reflexiones.pdf?sequence=1.

Soler, P., Trilla, J., Jiménez-Morales, M., \& Úcar, X. (2017). La construcción de un modelo pedagógico del empoderamiento juvenil: espacios, momentos y procesos. Pedagogía Social. Revista Interuniversitaria, 30, 25-40.

Suarez-Balcazar, Y., Orellana-Damacela, L., Portillo, N., Sharma, A., \& Lanum, M. (2003). Implementing an Outcomes Model in the Participatory Evaluation of Community Initiatives. Journal of Prevention \& Intervention in the Community, 26 (2), 5-20.

Úcar, X., (Coord.) Núñez, H., Paladines, M.B., Pescador, R. \& Múñoz, N. (2016). Evaluación Participativa del empoderamiento juvenil. Proceso de evaluación participativa con jóvenes del "casal de joves" de Badia del Valles. Informe de investigación. Diposit Digital de Documents. Universitat Autónoma de Barcelona. http://ddd.uab.cat/record/167221.

Úcar, X., (Coord.) Planas, A., Novella, A., Rodrigo-Moriche, P., Vila, C. , Núñez, H., Paladines, M.B., Páez de la Torre, S., Turón, N. Páez, J., \& Arenillas, M. (2016). Informe del proceso de evaluación participativa del empoderamiento juvenil con 4 grupos de jóvenes. Diposit Digital de Documents. Universitat Autónoma de Barcelona. http://ddd.uab. cat/record/167223.

Úcar, X., Heras, P., \& Soler, P. (2014). La evaluación participativa de acciones comunitarias como metodología de aprendizaje para el empoderamiento personal y comunitario: Estudio de casos y procesos de empoderamiento. Pedagogía Social. Revista Interuniversitaria, 2014, 24, 21-47 Retrieved from http://www.upo.es/revistas/index.php/pedagogia_social / doi: 10.7179/PSRI_2014.24.02.

Úcar, X., Jiménez-Morales, M., Soler, P., \& Trilla, J. (2016) “Exploring the conceptualization and research of empowerment in the field of youth". International Journal of Adolescence and Youth, doi:10.1080/02673843.2016.1209120.

Weaver, L., \& Cousins, B. (2004). Unpacking the participatory process. Journal of Multidisciplinary evaluation, 1, 19-40. Retrieved from http://www.astir.it/teseo/Download/Libanora/Unpacking\%2OParticipatory\%2OProcess_2002.pdf.

[EVALUACIÓN PARTICIPATIVA DEL EMPODERAMIENTO JUVENIL CON GRUPOS DE JÓVENES. ANÁLISIS DE CASOS] SIPS - PEDAGOGÍA SOCIAL. REVISTA INTERUNIVERSITARIA [(2017) 30, 67-80] TERCERA ÉPOCA 


\section{Notas}

'El equipo de investigación está compuesto por Pere Soler (IP), Jaume Trilla, Ana Novella, Asun Llena, Pilar Heras, Xavier Úcar, Héctor Núñez, Pilar Pineda, Íngrid Agud, Manel Jiménez, Alan Salvadó, Anna Planas, Sonia Páez, Judit Font, Narcís Turon y Myrte Monseny. Cuenta también con la colaboración de: Päivi Garriga, Genís Martín, Pilar Rodrigo, Anna Ciraso, Feliu Fusté, Ariadna Jordà, Ariadna Alonso y Mercè Gómez

2 Pueden ser respectivamente consultadas en los anexos 1 y 2.

${ }^{3}$ Esta definición, así como todo el trabajo de análisis teórico alrededor del concepto, puede verse en Úcar, Jiménez, Soler \& Trilla, 2016. En el primer artículo de este este mismo monográfico puede verse una versión mejorada y ampliada de dicho concepto. Ver Soler, Trilla, Jiménez-Morales \& Úcar, 2017.

${ }^{4}$ Esta batería, inicialmente de empoderamiento personal y comunitario, fue elaborada por el grupo de investigación en un proyecto previo de l+D+I (Ref. EDU2010-15122) (ver Soler, Planas, Ciraso-Calí \& Ribot-Horas, 2014). En el marco de la investigación actual, es adaptada para el empoderamiento juvenil y validada con académicos y profesionales especializados en juventud.

${ }^{5}$ Para trabajar la construcción del concepto de empoderamiento y las características de una persona empoderada se proponen tres técnicas que fomentan la deliberación y la reflexión emergente entre los jóvenes. Estás son: (1) La técnica de la flor. Invita a los participantes a identificar las palabras que se representan cuando piensan en el término "empoderamiento". (2) La técnica del retrato robot. Facilita que los jóvenes participantes representen sobre una figura humana todas aquellas características que asocian a una persona empoderada. (3) La recogida de definiciones e ideas sobre el empoderamiento en internet y entre sus familiares y personas de su barrio. Cada grupo escogió las dinámicas específicas a desarrollar.

\section{Anexo 1. Concepto}

“El empoderamiento juvenil puede ser un proceso o un resultado consecuencia de una interacción, más - menos negociada, entre las capacidades de acción de una persona joven y las opciones que le provee el medio físico y sociocultural en el que desenvuelve su vida. El término se refiere, de manera general, al crecimiento eficiente de la persona joven por medio de la superación de determinadas situaciones a través de la adquisición o desarrollo de competencias" (Úcar, Jiménez-Morales, Soler \& Trilla, 2016).

Anexo 2. Batería de Indicadores de Empoderamiento Juvenil

\begin{tabular}{|c|c|}
\hline Indicadores & Identificadores \\
\hline \multirow{6}{*}{ 1-Autoestima } & 1.1- Estar satisfecho con uno mismo \\
\hline & 1.2- Ser capaz para afrontar situaciones difíciles o adversas \\
\hline & 1.3- Ser capaz de mostrarse ante los demás \\
\hline & 1.4- Sentirse seguro con uno mismo \\
\hline & 1.5- Conocer las propias capacidades y reconocer los propios límites \\
\hline & 1.6- Sentirse reconocido por los demás \\
\hline \multirow{3}{*}{ 2- Responsabilidad } & 2.1- Asumir compromisos y tareas de forma voluntaria y realista \\
\hline & 2.2- Asumir funciones en los grupos y colectivos de los que se forma parte \\
\hline & 2.3- Ser capaz de compartir funciones y tareas \\
\hline \multirow{3}{*}{ 3- Eficacia } & 3.1- Ser capaz de tomar decisiones para conseguir los objetivos \\
\hline & 3.2- Ser metódico y constante en la realización de las tareas \\
\hline & 3.3- Conseguir los objetivos planteados \\
\hline
\end{tabular}




\begin{tabular}{|c|c|}
\hline Indicadores & Identificadores \\
\hline \multirow{2}{*}{ 4- Capacidad crítica } & 4.1- Ser capaz de analizar problemáticas o situaciones \\
\hline & 4.2- Tener un criterio propio en relación a problemáticas o situaciones \\
\hline \multirow{2}{*}{ 5- Autonomía } & 5.1- Tener iniciativa \\
\hline & 5.2- Ser capaz de elegir y actuar siguiendo las propias convicciones \\
\hline \multirow{4}{*}{ 6- Trabajo en equipo } & 6.1- Implicarse en el trabajo en equipo \\
\hline & 6.2- Ser capaz de ejercer funciones de liderazgo en el trabajo en equipo \\
\hline & 6.3- Ser capaz de comunicarse \\
\hline & 6.4- Ser capaz de negociar y consensuar \\
\hline \multirow{4}{*}{$\begin{array}{l}\text { 7- Identidad y Conocimiento } \\
\text { comunitario }\end{array}$} & 7.1- Conocer la historia y las dinámicas socioculturales de la comunidad \\
\hline & 7.2- Conocer los diferentes agentes y organizaciones de la comunidad \\
\hline & 7.3- Conocer los servicios, recursos y equipamientos del territorio \\
\hline & 7.4- Tener sentimiento de pertenencia \\
\hline \multirow{3}{*}{ 8- Metaprendizaje } & 8.1- Tener consciencia de haber adquirido o mejorado los propios conocimientos y capacidades \\
\hline & 8.2- Haber desarrollado la capacidad de aprender a aprender \\
\hline & 8.3- Tener conciencia del poder adquirido para actuar \\
\hline \multirow{2}{*}{ 9- Participación } & 9.1- Implicarse en acciones o proyectos \\
\hline & 9.2- Ser capaz de influenciar en su entorno \\
\hline
\end{tabular}

\section{CÓMO CITAR ESTE ARTÍ́CULO}

Úcar, X., Rodrigo, P., Planas, A., \& Novella, A. (2017). Evaluación participativa del empoderamiento juvenil con grupos de jóvenes. Análisis de casos. Pedagogía Social. Revista Interuniversitaria, 30, 67-80. DOI:10.7179/PSRI_2017.30.05.

\section{DIRECCIÓN COMPLETA DE LOS AUTORES}

Xavier Úcar: Universidad Autónoma de Barcelona. Dpto. Pedagogía Sistemática y Social. (España) Edifici G6 Campus de la UAB. 08193 Bellaterra (Cerdanyola del Vallès). Xavier.ucar@uab.cat

Anna Planas: Universitat de Girona (UdG). anna.planas@udg.edu

Ana Novella: Universidad de Barcelona (UB). anovella@ub.ed

Pilar Rodrigo Moriche: Universidad Autónoma de Madrid (UAM). pilar.rodrigo@uam.es

[EVALUACIÓN PARTICIPATIVA DEL EMPODERAMIENTO JUVENIL CON GRUPOS DE JÓVENES. ANÁLISIS DE CASOS] SIPS - PEDAGOGÍA SOCIAL. REVISTA INTERUNIVERSITARIA [(2017) 30, 67-80] TERCERA ÉPOCA 


\section{PERFIL ACADÉMICO}

Xavier Úcar: Catedrático de Pedagogía Social en Dpto. de Pedagogía Sistemática y Social de la Universidad Autónoma de Barcelona. Presidente de la "Sociedad Iberoamericana de Pedagogía Social" (SIPS) desde el 2012. Autor de más de un centenar de trabajos que incluyen libros, capítulos de libros y artículos en revistas científicas. La última publicación (2016) es una trilogía denominada "Pedagogías de lo social", que da título al primer libro y es complementada por "Relaciones socioeducativas. La acción de los profesionales" y "Pedagogía de la elección". Las tres editadas por "UOC Publishing" de Barcelona. http://uab.academia.edu/XavierUcar.

Anna Planas: Agregada interina en el Departamento de Pedagogía de la Universitat de Girona. Coordinadora del Grado en Educación Social desde de 2014. Trabajó como técnica de cultura y juventud, y coordinadora de proyectos educativos en el sector público, privado y en el tercer sector. Su investigación se centra en la animación sociocultural, la evaluación y las políticas y programas de juventud, de los cuales ha publicado decenas de artículos y capítulos de libro. Miembro de la Sociedad Iberoamericana de Pedagogía Social. http://www.udg.edu/personal/anna.planas.llado.

Ana Novella: Agregada interina en el Departamento de Teoría e Historia de la Educación de la Facultad de Educación de la Universidad de Barcelona. Integrante del Grupo de Investigación en Educación Moral (GREM). Los temas investigación que desarrollo son la participación en la escuela, la participación ciudadana, los consejos infantiles y el empoderamiento juvenil. Co-Investigadora del proyecto "Los consejos infantiles. Nuevas formas de participación política y cívica de los niños y las niñas de Cataluña" (Recercaixa, 2014). Algunas de las publicaciones recientes son: Infant, participació i ciutat. El consell d'infants, un exercici de ciutadania. Barcelona (Horsori, 2013); Participación y construcción de la ciudadanía. (en Novella et al., Graó 2014). http://www. ub.edu/GREM/professorat/ana-maria-novella-camara/.

Pilar Rodrigo Moriche: Profesora en la Facultad de Formación del Profesorado y Educación de la Universidad Autónoma de Madrid (UAM) desde 2011; Profesora colaboradora en el Dpto. de Ta de la Educación y Pedagogía Social de la UNED. Maestra de educación infantil, pedagoga, máster TIC en Educación, y Doctora en Ciencias de la Educación por la UAM. Miembro del Grupo de Investigación para la Inclusión Social y Laboral de Personas con Discapacidad Intelectual reconocido por la UAM, de la Red OcioGune, y de la Red de Excelencia de Estudios sobre Juventud y Sociedad. goo.gl/shoPrT. 